Research Paper

\title{
TMPRSS6 rs85579I Polymorphism Influences the Susceptibility to Iron Deficiency Anemia in Women at Reproductive Age
}

\author{
Sung-Nan Pei ${ }^{1,2,3}$, Ming-Chun Ma1, Huey-Ling You ${ }^{2,4}$, Hung-Chun Fu' ${ }^{2,5}$, Ching-Yuan Kuo', Kun-Ming \\ Rau $^{1,2}$, Ming-Chung Wang1,2, Chien-Te Lee ${ }^{2,6}$ \\ 1. Division of Hema-Oncology, Department of Internal Medicine, Kaohsiung Chang Gung Memorial Hospital, Kaohsiung, Taiwan \\ 2. Chang Gung University College of Medicine, Taoyuan, Taiwan \\ 3. Graduate Institute of Clinical Medical Sciences, Chang Gung University College of Medicine, Taoyuan, Taiwan \\ 4. Department of Laboratory Medicine, Kaohsiung Chang Gung Memorial Hospital \\ 5. Department of Obstetrics and Gynecology, Kaohsiung Chang Gung Memorial Hospital \\ 6. Division of Nephrology, Department of Internal Medicine, Kaohsiung Chang Gung Memorial Hospital, Kaohsiung, Taiwan
}

$\triangle$ Corresponding author: Chien-Te Lee, MD, PhD, Department of Internal Medicine, Kaohsiung Chang Gung Memorial Hospital, 123, Ta-Pei Road, Niao-Sung District, Kaohsiung City 833, Taiwan. Tel: +886 7 7317123; Fax: +886 7 7322402; E-mail: chientel@gmail.com

( ) Ivyspring International Publisher. This is an open-access article distributed under the terms of the Creative Commons License (http://creativecommons.org/ licenses/by-nc-nd/3.0/). Reproduction is permitted for personal, noncommercial use, provided that the article is in whole, unmodified, and properly cited.

Received: 2014.01.14; Accepted: 2014.03.14; Published: 2014.04.16

\begin{abstract}
Background: Genome-wide-association studies have identified the TMPRSS6 polymorphism rs85579I has the strongest association with red blood cell indices or iron parameters in general population. Whether this genetic variant influences the susceptibility of iron deficiency anemia (IDA) in women with menstruation has not been well studied.

Methods: In this case-control study, we enrolled 67 women with IDA and 107 healthy volunteers, and analyzed their complete blood counts, rs85579I genotypes, and menstrual amounts. Menstrual blood loss was evaluated with a pictorial blood-loss assessment chart.

Results: There were significantly fewer rs85579 $C$ homozygotes in the IDA group than in the healthy group (II.9\% vs. $25.2 \%, p=0.03)$. The odds ratio $(O R)$ of $C$ homozygotes having IDA versus non-CC subjects having IDA was $0.4(95 \% \mathrm{Cl}, 0.17-0.95, \mathrm{p}=0.04)$. When the analysis was confined to study subjects with menorrhagia, this difference became more prominent $(9.6 \%$ vs. $28.6 \%, p=0.01 ; O R, 0.27,95 \% \mathrm{Cl}, 0.09-0.77, p=0.01$ ). For women with non-CC genotypes, there was an inverse correlation between hemoglobin levels and menstrual loss $(P<0.00 I)$; however, this association was not found for those with genotypes $C C(p=0.15)$.

Conclusions: Our study suggests homozygosity for TMPRSS6 rs85579I C genotype has a protective role against IDA in women at reproductive age, especially in those with menorrhagia.
\end{abstract}

Key words: iron deficiency anemia, menorrhagia, single nucleotide polymorphism, rs855791, transmembrane protease serine 6 (TMPRSS6)

\section{Introduction}

Iron deficiency is the most common nutritional disorder worldwide. As well as affecting a large number of children and women in non-industrialized countries, it is the only nutrient deficiency which is also significant prevalent in many industrialized nations [1]. Menstruating women have been recognized to be among the most likely individuals to develop iron deficiency anemia (IDA) and its prevalence is $6-22 \%$ in developed countries [2-5]. Even though menorrhagia serve as a major contributing factor of IDA in this population, it is not uncommon that women with heavy menstrual loss have normal he- 
moglobin $(\mathrm{Hb})$ levels and adequate iron stores [2]. Individual susceptibility to IDA varies. However, whether genetic variants play a role in IDA pathogenesis remains unclear.

Hepcidin is the core of iron metabolism and is tightly regulated by several mediators [6]. Matriptase- 2 is an important one and down regulates hepcidin expression through cleaving membrane-bound hemojuvelin, which can enhance hepcidin transcription [6]. Complete loss of function mutation of matriptase-2 leads to a rare disease, iron-refractory iron deficiency anemia (IRIDA) [7, 8]. Patients with IRIDA have high serum hepcidin levels and cannot absorb iron from intestine. It is of interest that whether genetic variants with incomplete loss of function of matriptase- 2 will be associated with IDA in general population.

Recently, several genome-wide association studies identified several single nucleotide polymorphisms (SNPs) that influence blood cell phenotypes [9-13]. Among these SNPs, rs855791 has the strongest association with red blood cell indices and iron parameters in general population [12,13]. This SNP is located in the functional part of TMPRSS6 and the rs855791 (2321 C>T) causes a nonsynonymous substitution that reduces the ability of the enzyme to inhibit hepcidin transcription [14]. To investigate the role of this genetic variant rs855791 on the susceptibility of IDA in menstruating women, we conducted this case-control study.

\section{Materials and Methods}

\section{Study population}

Female patients with microcytic anemia were screened at hematologic clinics for IDA. IDA was defined as a hemoglobin level $<12 \mathrm{~g} / \mathrm{dL}$ and a ferritin level $<10 \mathrm{ng} / \mathrm{mL}[2,15]$. The evaluation for IDA included a physical examination, history taking, renal function test, and stool occult blood test. For those without an adequate response to iron replacement (oral elemental iron $300 \mathrm{mg}$ /day for 2-4 weeks), $\mathrm{Hb}$ electrophoresis and brilliant cresyl blue staining tests were used to exclude thalassemia. Healthy women with regular menstruation were enrolled as the control group; most of them were staff in our institute. Complete blood counts and ferritin levels were measured and only those with normal $\mathrm{Hb}$ and ferritin levels were included in the analysis. An in-person questionnaire was administered to every subject in this study, which included information on menstrual, medical and surgical history. The exclusion criteria for both groups included gastrointestinal bleeding, thalassemia, renal insufficiency, menopause, and history of gastrointestinal resection. This study was per- formed in accordance with the declaration of Helsinki, and approved by the Ethics Committee of Chang-Gung Memorial Hospital (99-0482B) and informed consent was obtained from all participants.

\section{Assessment of menstrual bleeding}

Menstrual blood loss was evaluated with the pictorial blood-loss assessment chart (PBAC) [16], a semi-quantitative method used to estimate menstrual amount and has been validated in many studies [17-19]. Menorrhagia was defined as a PBAC score of more than 100 during one menstrual period, which corresponds to a blood loss of more than $80 \mathrm{~mL}$ [16].

\section{Red blood cell parameters and ferritin levels}

Red blood cell numbers, $\mathrm{Hb}$, hematocrit, mean corpuscular volume, mean corpuscular hemoglobin, mean corpuscular hemoglobin concentration, red cell distribution width and platelet counts were determined by the XE-2100 system (Sysmex Co, Kobe, Japan). Serum ferritin levels were determined using a commercial EIA kit (Advia Centaur, Siemens, NY).

\section{Genotyping}

Genomic DNA was extracted from peripheral leukocytes using the Genomic DNA Purification Kit (Gentra Systems, Inc. Chicago, IL, USA). The TMPRSS6 rs855791 C>T polymorphism was determined by polymerase chain reaction (PCR) and restriction fragment length polymorphism (RFLP)(Figure 1). The primers used were $5^{\prime}$-tag aga aca ggg gct cca gg- $3^{\prime}$ (forward) and $5^{\prime}$-atg tgg gca gca tcc ttt c-3' (reverse). The reaction conditions for amplification were as follows: $95^{\circ} \mathrm{C}$ for $5 \mathrm{~min} ; 30$ cycles of $95^{\circ} \mathrm{C}$ for $45 \mathrm{~s}, 63^{\circ} \mathrm{C}$ for $45 \mathrm{~s}$, and $72^{\circ} \mathrm{C}$ for $45 \mathrm{~s}$; and a final extension at $72^{\circ} \mathrm{C}$ for $5 \mathrm{~min}$. Following amplification, the PCR products ( $249 \mathrm{bp}$ ) were digested with the restriction endonuclease Stu I (New England Biolabs, Inc. Hitchin, Herts, UK). Genotype was determined by fragment size, and $10 \%$ of the samples were directly sequenced to confirm the genotyping results.

\section{Statistical analysis}

Continuous variables are presented as mean \pm standard deviation; categorical variables are presented as numbers and percentages. Categorical variables were analyzed by Chi-square or Fisher's exact tests. Student's t-test was used to examine continuous variables with normal distributions. Linear correlation and regression was used to evaluate the correlations between age, $\mathrm{Hb}$ levels and menstrual amounts. Odds ratio (OR) and confidence interval (CI) were tested by binary logistic regression. A p-value of less than 0.05 was considered statistically significant. 
A

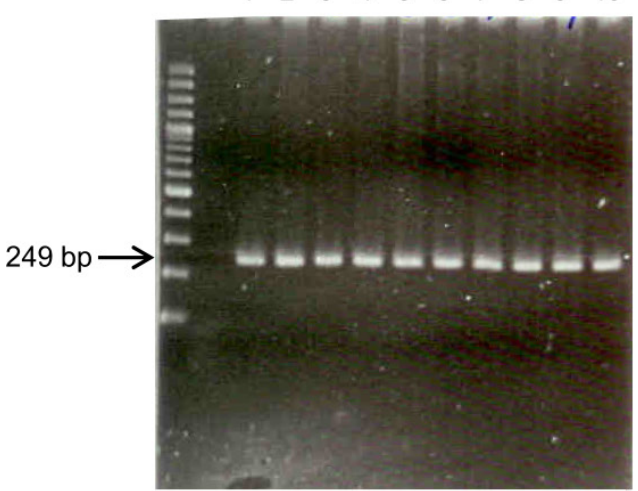

$\mathrm{B}$

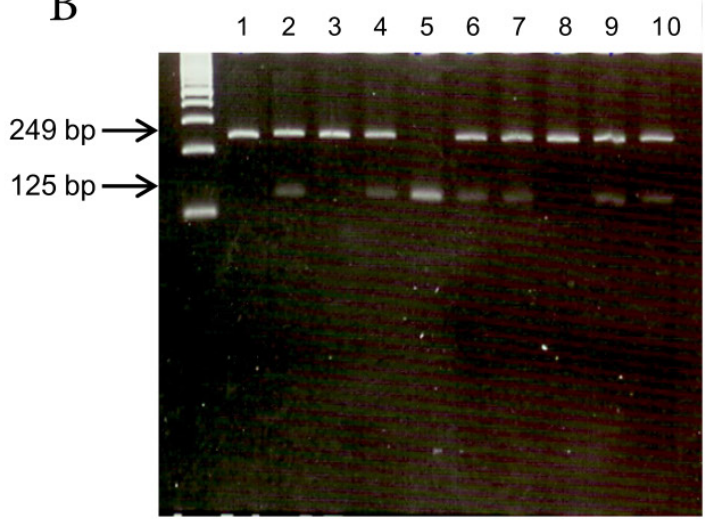

Figure I. Genotyping of TMPRSS6 rs85579I by (A) PCR (products $249 \mathrm{bp}$ ) and (B) restriction fragment length polymorphism (RFLP). The PCR products were digested with Stu I endonuclease. Single band at 249 bp represented C homozygous; single band at I 25 bp suggested T homozygous, and two bands represented heterozygous.

Table I. Laboratory data and menstruation amount of both groups

\begin{tabular}{llll}
\hline & Control group $(\mathrm{n}=107)$ & IDA group $(\mathrm{n}=67)$ & $P$ value \\
\hline Age, $\mathrm{y}$ & $32.7+/-6.2$ & $39.5+/-9.2$ & $<0.001$ \\
Red blood cells, million/ $\mu \mathrm{L}$ & $4.42+/-0.28$ & $4.21+/-0.59$ & 0.01 \\
Hemoglobin, g/dL & $13.1+/-0.7$ & $8.5+/-2.1$ & $<0.001$ \\
Hematocrit, $\%$ & $38.8+/-4.3$ & $29.2+/-5.5$ & $<0.001$ \\
Mean corpuscular volume, fL & $88.7+/-3.14$ & $69.0+/-7.9$ & $<0.001$ \\
Mean corpuscular hemoglobin, pg/cell & $29.6+/-1.3$ & $20.1+/-3.7$ & $<0.001$ \\
Mean corpuscular hemoglobin concentration, g/dL & $33.4+/-0.9$ & $29.0+/-2.4$ & $<0.001$ \\
Red blood cell width, \% & $12.8+/-0.5$ & $18.0+/-3.4$ & $<0.001$ \\
Platelet, $1000 / \mu \mathrm{L}$ & $265.0+/-54.6$ & $344.2+/-105.6$ & $<0.001$ \\
Ferritin, $\mathrm{ng} / \mathrm{mL}$ & $60.0+/-46.7$ & $5.5+/-5.0$ & $<0.001$ \\
Pictorial blood-loss assessment chart score & $185.2+/-193.6$ & $362.5+/-352.4^{\mathrm{b}}$ & $<0.001$ \\
\hline
\end{tabular}

a Data are expressed as mean $+/$ - standard deviation; ${ }^{b} \mathrm{n}=65$.

\section{Results}

A total of 67 IDA subjects and 107 healthy women were eligible for analysis. Characteristics of both groups are shown in Table 1. IDA patients were significantly older than control subjects $(39.5 \pm 9.2$ vs. $32.7 \pm 6.2$ years; $p<0.001$ ). All red blood cell indices and platelet counts differed significantly between two groups (Table 1). PBAC scores were not available for 2 IDA subjects. The PBAC scores of the 65 IDA patients were significantly higher than those of the 107 healthy controls (362.5 \pm 352.4 vs. $185.2 \pm 193.6 ; p<0.001)$. Menorrhagia (PBAC score $>100$ ) was more common in IDA patients than in the control group $(80.0 \%$ vs. $65.4 \%, \mathrm{p}=0.04)$.

The frequency distribution of the rs855791 TT, $T C$, and CC genotypes in both groups showed borderline difference $(p=0.06$; Table 2$)$. However, the proportion of the $C$ homozygotes was significantly lower in IDA patients $(11.9 \%$ vs. $25.2 \%, p=0.03$; Figure 2). The odds ratio (OR) of $C$ homozygotes having IDA versus those with TX (TT and TC) genotypes having IDA was $0.4(95 \% \mathrm{CI}, 0.17-0.95, \mathrm{p}=0.04)$. The
$C$ allele frequencies in the IDA and control group did not differ $(43.3 \%$ vs. $48.6 \%$, $\mathrm{p}=0.33)$.

Table 2. TMPRSS6 rs85579I genotypes distribution between IDA and control groups

\begin{tabular}{lllll}
\hline \multicolumn{5}{l}{ TMPRSS6 rs855791 } \\
\hline & CC & TC & TT & p-value \\
$\begin{array}{l}\text { Control group } \\
\text { n=107 }\end{array}$ & $27(25.2 \%)$ & $50(46.7 \%)$ & $30(28.0 \%)$ & 0.06 \\
$\begin{array}{l}\text { IDA group } \\
\text { n=67 }\end{array}$ & $8(11.9 \%)$ & $42(62.7 \%)$ & $17(25.4 \%)$ & \\
\hline
\end{tabular}

IDA, iron deficiency anemia.

We further performed subgroup analysis based on menorrhagia (PBAC score > 100). For menorrhagic subjects, the frequency distribution of the 3 genotypes of the rs855791 SNP differed significantly in both groups ( $p=0.02$; Table 3 ). The frequency of the CC genotype was much lower in the IDA group $(9.6 \%$ vs. $28.6 \%, p=0.01$, Figure 3a). Menorrhagic women with homozygous $\mathrm{C}$ genotype have lower risk to have IDA than those with $T X$ genotypes $(\mathrm{OR}=0.27,95 \% \mathrm{CI}$, 0.09-0.77, $p=0.01)$. However, for those without 
menorrhagia, no difference between IDA and healthy women was observed in the frequency distribution of $T T, T C$, and CC genotypes ( $p=0.91$; Table 3 ) or of the CC and TX genotypes (OR $=0.78,95 \% \mathrm{CI}, 0.14-4.34, \mathrm{p}$ $=0.78$; Figure $3 \mathrm{~b}$ ). The frequency of the $\mathrm{C}$ allele did not differ between the groups whether or not the study subjects had menorrhagia (for menorrhagic subjects, $42.3 \%$ vs. $50.7 \%, p=0.19$; for non-menorrhagic subjects, $46.2 \%$ vs. $44.6 \%, p=0.89$ ).

Then, we pooled data from both groups, and analyzed the correlation between $\mathrm{Hb}$ levels and menstrual blood loss (PBAC scores). For women with genotypes $T X$, there was a strong inverse correlation between $\mathrm{Hb}$ levels and menstrual amounts; the equation used was $\mathrm{Hb}=11.95-0.003 \times$ PBAC score $\left(\mathrm{R}^{2}=\right.$ $0.135, \mathrm{p}<0.001)$. In contrast, the correlation was not statistically significant for those with $C$ homozygotes $\left(R^{2}=0.062, p=0.15\right)$. The results are also shown in Figure 4. Because subjects in the IDA group were older and had more menstrual blood loss than the control group, we therefore analyzed the correlation between age and PBAC scores in all subjects. We found that older women had more menstrual blood loss ( $p=0.002$, data not shown). This result explained the difference in the age distribution between two groups.
Finally, we investigated the influence of rs855791 on RBC parameters, ferritin levels, and $\log$ (ferritin) value based on the control group data. However, we did not find a difference between the genotypes in our study (data not shown).

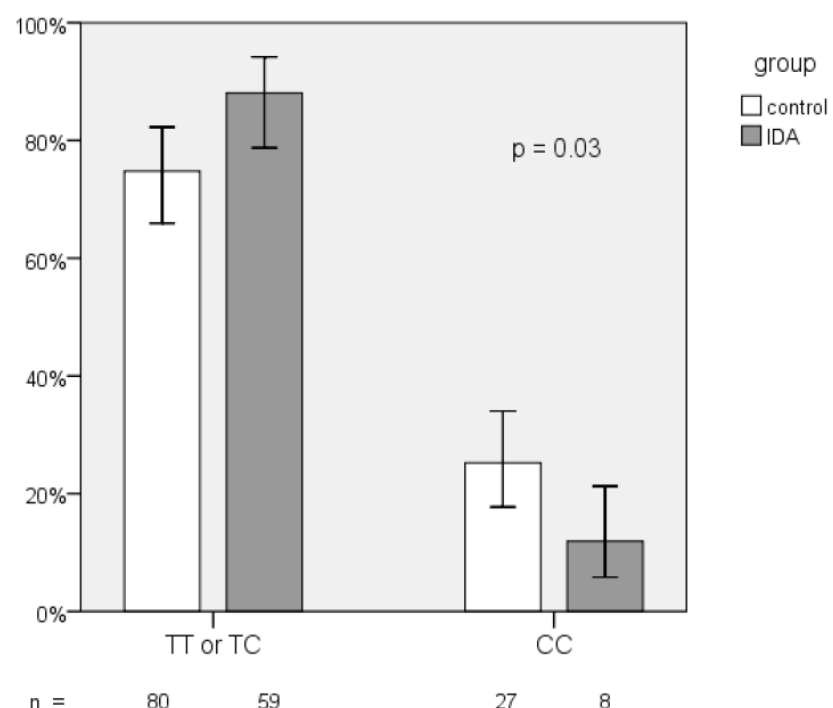

Figure 2. Frequency distribution of TMPRSS6 rs85579I genotypes between IDA and control group

Table 3. Subgroup analysis of TMPRSS6 rs85579I genotypes according to menorrhagia

\begin{tabular}{lllllllll}
\hline \multicolumn{2}{l}{ Menorrhagia (PBAC > 100) } & \multicolumn{5}{l}{ Non-menorrhagia (PBAC < 100) } \\
\hline & TMPRSS6 rs855791 & & & \multicolumn{3}{l}{ TMPRSS6 rs855791 } \\
\hline
\end{tabular}

IDA, iron deficiency anemia; PBAC pictorial blood-loss assessment chart.

A

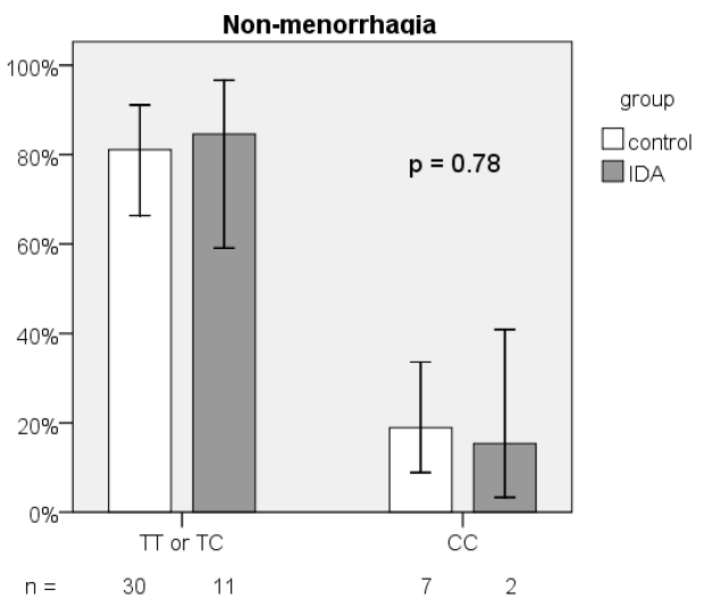

B

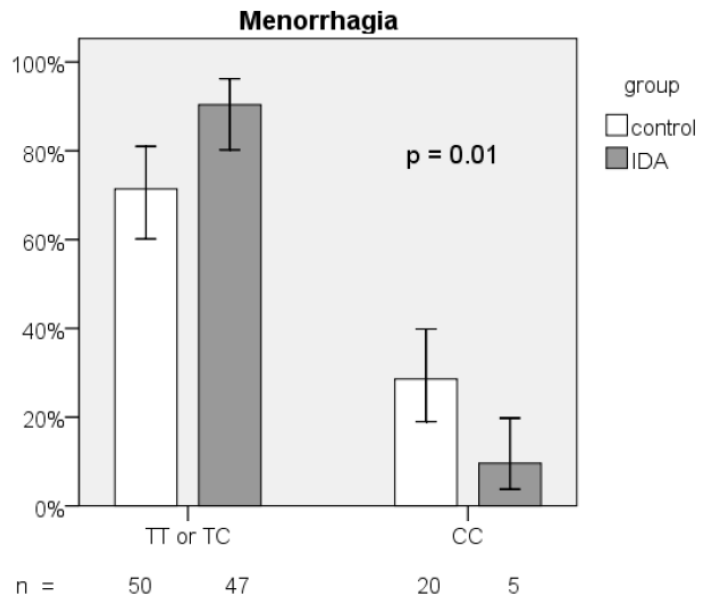

Figure 3. Frequency distribution of TMPRSS6 rs85579I genotypes between IDA and control groups according to (A) non-menorrhagia and (B) menorrhagia. 
A

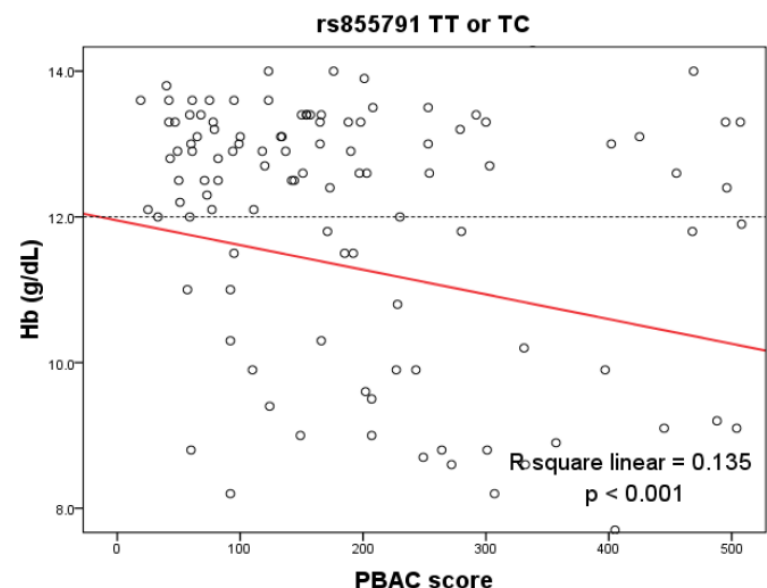

B

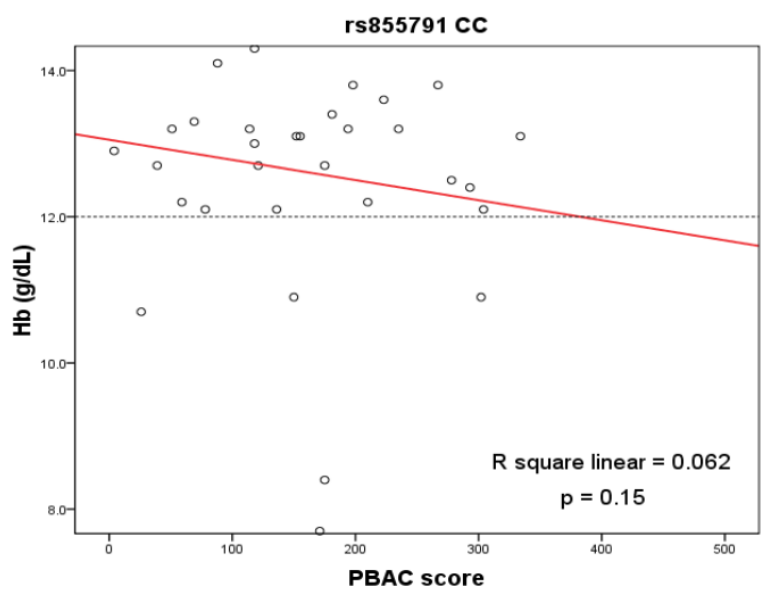

Figure 4. Linear correlation between menstrual amounts and hemoglobin levels were analyzed according to genotypes of TMPRSS6 rs85579I (A) TX (TT or TC) and (B) CC. There was an inverse correlation for women with genotypes TX, but not for those with genotype CC. PBAC, pictorial blood-loss assessment chart.

\section{Discussion}

In this study, we evaluated whether the TMPRSS6 rs855791 polymorphism influencing IDA susceptibility in menstruating women. There were fewer women with CC genotype in the IDA group and this difference became more obvious when the analysis was confined to those with menorrhagia. This result suggested that rs855791 $C$ homozygotes have a lower risk to have IDA, especially those with menorrhagia. Then, we explored the influence of this SNP on menstrual amount effect to IDA. For women with genotypes $T X, \mathrm{Hb}$ levels were inversely correlated with the PBAC scores. This result was compatible with the general concept that more menstrual loss would result in greater iron loss and subsequent IDA. However, this correlation was not significant in the $C$ homozygotes. This provides evidence that rs855791 C homozygotes serve a genetic protective factor against IDA. For the first time, we demonstrate that the higher ferritin levels of 736A homozygotes in the general population will transform into the lower susceptibility to IDA in women, especially for those with menorrhagia [13].

In our study, menorrhagia is still the major cause of IDA in reproductive-age women [2, 20]. Menstrual amount increased with age and the mean age of IDA group was older than control group; these phenomena were in line with previous reports the prevalence of menorrhagia increases with age, as does the risk of IDA [21]. However, the association between menstrual blood loss and IDA was no more significant for women with CC genotype. Addition to well-known risks contributing to IDA in this population, such as vegetarian and gastrointestinal bleeding, our result demonstrates that a genetic factor can also affect the susceptibility of menorrhagia-associated IDA.

Our study also provided the first report of rs855791 allelic frequency in Taiwan, located in East Asia. The $C$ allele frequency in our control group was $48.6 \%(104 / 214)$ which is similar to that in European epidemiologic studies (approximately 50\%) and higher than that in reported Asian countries (39-47\%) [22]. However, the $C$ allele frequency is reported to be 82 to $93 \%$ in African population; this high prevalence may confer an advantage of enhanced iron absorption during food shortages or provide some protection against parasites that invade RBCs, such as malaria [22].

The SNP rs855791 locates in the catalytic domain of matriptase-2. Nai et al. recently showed that the rs855791 C genotype inhibits hepcidin more efficiently than the $T$ genotype in vitro and then confirmed that $C$ homozygous individuals have lower serum hepcidin levels and higher transferrin saturation than those with $\mathrm{T}$ homozygotes in the general population [22]. However, they excluded iron-deficient subjects, a population of great interest to us. Very recently, a population-based study showed the TMPRSS6 genetic variants, including rs855791, are associated with IDA susceptibility in elderly women [23]. The OR of the protective allele was relative insignificant (OR, 0.56; $95 \% \mathrm{CI}, 0.51-0.63$ ) based on their large sample size (n $=2,139$ ); and the reason may be that most of their study population was post-menopausal and at relatively low risk for IDA. In our study, we selected a population at high-risk for IDA, that is, menstruating women. With the enhancement of menstrual blood loss, the role of this SNP would be highlighted. Furthermore, most of the subjects in our study (>98\%) 
have PBAC scores and these data allowed us to perform additional analysis according to menstrual amount. Then, we could clarify the protective role of this polymorphism in menorrhagic women.

We did not measure hepcidin levels in our subjects. Therefore, we could not analyze the correlation between rs855791 and hepcidin levels. According to Nai's study, $C$ homozygotes have lower hepcidin levels than $T$ homozygotes in the general population [22]. We speculate that the protective role of $C$ homozygous rs855791 in our study is due to decreased hepcidin levels. Besides, because mammals lack a regulated pathway for iron excretion [6], it is likely that women with the CC genotype increase the iron absorption from the intestine through decreased hepcidin production and then can offset the menstrual losses. From clinical aspect, iron replacement till 3 to 6 months after relieving anemia is recommended [15]. Then, how to prevent relapse may be determined by this genetic variant. For those with $C C$ genotypes, diet modification may be enough to keep iron balance; otherwise, long-term iron replacement till menopause may be considered.

In conclusion, our data showed that TMPRSS6 rs855791 CC genotype is less frequent in reproductive-age women with IDA than in healthy women. Menorrhagia is the major risk factor for IDA in menstruating women with TX genotypes, but not for those with CC genotype. Homozygosity for rs855791 C genotype plays a protective role against IDA, especially for those with menorrhagia.

\section{Acknowledgements}

This work was supported by grants from Chang Gung Memorial Hospital (grant no. CMRPG 891201).

\section{Competing Interests}

The authors have declared that no competing interest exists.

\section{References}

1. [Internet] World Health Organization. Iron deficiency anemia: assessment, prevention, and control. http://www.who.int/nutrition/publications/ en/ida assessment prevention control.pdf

2. Killip S, Bennett JM, Chambers MD. Iron deficiency anemia. American family physician. 2007; 75: 671-8.

3. Clark SF. Iron deficiency anemia. Nutrition in clinical practice : official publication of the American Society for Parenteral and Enteral Nutrition. 2008; 23: $128-41$.

4. McClung JP, Marchitelli LJ, Friedl KE, Young AJ. Prevalence of iron deficiency and iron deficiency anemia among three populations of female military personnel in the US Army. Journal of the American College of Nutrition. 2006; 25: 64-9.

5. Looker AC, Dallman PR, Carroll MD, Gunter EW, Johnson CL. Prevalence of iron deficiency in the United States. JAMA : the journal of the American Medical Association. 1997; 277: 973-6.

6. Hentze MW, Muckenthaler MU, Galy B, Camaschella C. Two to tango: regulation of Mammalian iron metabolism. Cell. 2010; 142: 24-38.

7. Melis MA, Cau M, Congiu R, Sole G, Barella S, Cao A, et al. A mutation in the TMPRSS6 gene, encoding a transmembrane serine protease that suppresses hepcidin production, in familial iron deficiency anemia refractory to oral iron. Haematologica. 2008; 93: 1473-9.
8. Finberg KE, Heeney MM, Campagna DR, Aydinok Y, Pearson HA, Hartman $\mathrm{KR}$, et al. Mutations in TMPRSS6 cause iron-refractory iron deficiency anemia (IRIDA). Nat Genet. 2008; 40: 569-71.

9. Kullo IJ, Ding K, Jouni H, Smith CY, Chute CG. A genome-wide association study of red blood cell traits using the electronic medical record. PLoS One. 2010; 5: e13011.

10. Soranzo N, Spector TD, Mangino M, Kuhnel B, Rendon A, Teumer A, et al. A genome-wide meta-analysis identifies 22 loci associated with eight hematological parameters in the HaemGen consortium. Nat Genet. 2009; 41: 1182-90.

11. Ganesh SK, Zakai NA, van Rooij FJ, Soranzo N, Smith AV, Nalls MA, et al. Multiple loci influence erythrocyte phenotypes in the CHARGE Consortium. Nat Genet. 2009; 41: 1191-8.

12. Chambers JC, Zhang W, Li Y, Sehmi J, Wass MN, Zabaneh D, et al Genome-wide association study identifies variants in TMPRSS6 associated with hemoglobin levels. Nat Genet. 2009; 41: 1170-2.

13. Benyamin B, Ferreira MA, Willemsen G, Gordon S, Middelberg RP, McEvoy $\mathrm{BP}$, et al. Common variants in TMPRSS6 are associated with iron status and erythrocyte volume. Nat Genet. 2009; 41: 1173-5.

14. Nai A, Pagani A, Silvestri L, Camaschella C. Increased susceptibility to iron deficiency of Tmprss6-haploinsufficient mice. Blood. 2010; 116: 851-2.

15. Andrews NC. Iron deficiency and related disorders. Wintrobe's Clinical Hematology 12th ed. Philadelphia, PA: Lippincott Williams \& Wilkins. 2009: 810-34.

16. Higham JM, O'Brien PM, Shaw RW. Assessment of menstrual blood loss using a pictorial chart. British journal of obstetrics and gynaecology. 1990; 97: 734-9.

17. Donnez J, Tatarchuk TF, Bouchard P, Puscasiu L, Zakharenko NF, Ivanova T, et al. Ulipristal acetate versus placebo for fibroid treatment before surgery. $\mathrm{N}$ Engl J Med. 2012; 366: 409-20.

18. Fu HC, Huang KH, Tseng CW, Liang HM, Lin H, Chou YJ, et al. Comparison of clinical outcomes and spectral Doppler indices of uterine and ovarian stromal arteries in women undergoing myomectomy with or without hypogastric arterial ligation. Ultrasound in obstetrics \& gynecology : the official journal of the International Society of Ultrasound in Obstetrics and Gynecology. 2006; 28: 831-6.

19. Philipp CS, Faiz A, Dowling N, Dilley A, Michaels LA, Ayers C, et al. Age and the prevalence of bleeding disorders in women with menorrhagia. Obstet Gynecol. 2005; 105: 61-6.

20. Oehler MK, Rees MC. Menorrhagia: an update. Acta obstetricia et gynecologica Scandinavica. 2003; 82: 405-22.

21. Marret H, Fauconnier A, Chabbert-Buffet N, Cravello L, Golfier F, Gondry J, et al. Clinical practice guidelines on menorrhagia: management of abnormal uterine bleeding before menopause. European journal of obstetrics, gynecology, and reproductive biology. 2010; 152: 133-7.

22. Nai A, Pagani A, Silvestri L, Campostrini N, Corbella M, Girelli D, et al. TMPRSS6 rs855791 modulates hepcidin transcription in vitro and serum hepcidin levels in normal individuals. Blood. 2011; 118: 4459-62.

23. An P, Wu Q, Wang $\mathrm{H}$, Guan $\mathrm{Y}$, Mu M, Liao $\mathrm{Y}$, et al. TMPRSS6, but not TF, TFR2 or BMP2 variants are associated with increased risk of iron-deficiency anemia. Human Molecular Genetics. 2012; 21: 2124-31. 